\title{
Deterrents to Viewing Indecent Images of Children Online: A Protocol for a Meta-Narrative Review
}

\author{
Emily F. Newman ${ }^{1 *}$, Ethel Quayle ${ }^{1}$, Laura A. Cariola ${ }^{1}$, Karen Cooper ${ }^{1}$, Nikolaos \\ Koukopoulos $^{1}$, Klaus M. Beier ${ }^{2}$, Tom Squire ${ }^{3}$, Richard Wortley ${ }^{4}$ \\ ${ }^{1}$ School of Health in Social Science, University of Edinburgh, Edinburgh, UK \\ ${ }^{2}$ Department of Health and Human Sciences, Institute of Sexology and Sexual Medicine, \\ Universitätsmedizin, Berlin, Germany \\ ${ }^{3}$ The Lucy Faithfull Foundation, Epsom, UK \\ ${ }^{4}$ UCL Jill Dando Institute of Security and Crime Science, London, UK
}

\begin{abstract}
Background: Evidence indicates that the viewing of indecent images of children (IIOC) has increased due to the emergence of, and increased access to, the Internet. Over the last 20 years there has been a high level of collaboration between various stakeholders to prevent and restrict access to IIOC. Nevertheless, there is no comprehensive review of these approaches or their effect as deterrents to viewing IIOC.
\end{abstract}

Methods/Design: This paper presents a protocol for a meta-narrative review of the deterrents to viewing indecent images of children (IIOC) online. The meta narrative review methodology allows researchers to systematically identify and review different understandings, or narratives, of a subject across a range of disciplines, with the aim of synthesising this diverse literature and providing recommendations to researchers, practitioners and policymakers. We outline the authors' approach to the review, focusing on six methodological steps: planning the review scope, questions and outputs, systematic searching of academic and grey literature, mapping the identified articles according to disciplines and their narratives, appraising the evidence within narratives, then synthesising the conceptualisations and evidence from the different disciplines to generate overarching narratives and recommendations to stakeholders.

Discussion: The discussion considers the anticipated benefits and challenges of conducting the review, and makes recommendations for other researchers embarking on this type of review.

Keywords: indecent images of children; child pornography; internet; deterrence; metanarrative review

\footnotetext{
* Correspondence to Dr. Emily Newman, School of Health in Social Science, The University of Edinburgh, Teviot Place, Edinburgh, EH8 9AG, UK. Tel: +44 (0) 131651 3945. Email: emily.newman@ed.ac.uk
} 


\section{Background}

Evidence indicates that the viewing of indecent images of children (IIOC) has increased due to the emergence of, and increased access to, the Internet (Wolak, Finkelhor \& Mitchell, 2012). In Schuijer and Rossen's (1992) study of the trade of IIOC, it was calculated that between 1972 and 1984 there were 508 magazines depicting boys and 288 depicting girls in IIOC. As a comparison, in 2016 the UK Internet Watch Foundation (IWF) reported that in that year 57,335 URLs (based on reports by members of the public as well as proactive searching by the IWF) were confirmed as containing child sexual abuse imagery, having links to the imagery, or advertising it. It is impossible to determine exactly how many people are accessing IIOC online because many offences are undetected (Elliott \& Beech, 2009). Nevertheless, an increase in the number of online offences recorded suggests that the greater accessibility to IIOC afforded by the internet has created a significant global problem in terms of creating and viewing IIOC.

There is evidence of the effectiveness of police activity in detection of IIOC viewers, particularly pro-active policing (e.g. Operation Latisse; BBC News, 2016). However, evidence from the US has demonstrated that arrests for the possession of IIOC increased by one third from 2006 to 2009, and also in the proceeding period between 2000 and 2006 (Wolak, Finkelhor \& Mitchell, 2012). This is despite growing legislative, judicial, law enforcement and media attention as well as in the context of increased sanctions in the US, where, for a first-time offender, a conviction for possession of "child pornography" would carry a sentence of 0-10 years in Federal custody. Therefore, legal consequences might not be adequately deterring the production, distribution and possession of this material (Henzey, 2011). Over the last 20 years there has been a high level of collaboration between various stakeholders to prevent and restrict access to IIOC, including law enforcement, industry, child protection services, prison and probation services, educational organisations, families, financial services and civil society, alongside charities, government and non-government organisations. This cross-disciplinary, multi-stakeholder approach has served to increase awareness, limit access, reduce content and offer support to those worried about their thoughts and compulsions and help manage those who have viewed IIOC. Nevertheless, there is no comprehensive review of these approaches or their effect as deterrents to viewing IIOC.

One of the foreseeable difficulties in conducting a review of deterrents is that there is not a single, universal understanding, or application, of the word deterrence. In relation to viewing online IIOC, deterrence could refer to preventing someone from searching for or viewing the images in the first place, or could mean stopping a behaviour from recurring, which may or may not precede detection from law enforcement agencies. Deterrence can also be considered with respect to the individual (the viewer) and the environment (both virtual and offline). The individual's psychological characteristics are likely to influence whether such material is desired and sought out, and subsequently sought again. Indeed, a body of research has considered the psychological profile of those viewing online IIOC, in part driven by a need to understand the risk of offences escalating to contact sexual offences against children, or whether those viewing online IIOC represent a separate offender typology. An investment in answering these questions is demonstrated by the development of specific risk appraisal tools, such as KIRAT (Kent Internet Risk Assessment Tool; CEOP, 2012) and CPORT (Child Pornography Offender Risk Tool; Seto \& Eke, 2015), and investigations of the psychological characteristics of image-related offenders (Elliott, Beech \& MandevilleNorden, 2013; Quayle \& Newman, 2015; Seto, Hanson \& Babchishin, 2011). This research has clear implications for deterrence in relation to developing appropriate treatment programmes to prevent offending and recidivism, and indeed there is emerging evidence of the effects of the Dunkelfeld Project (Beier et al., 2015), the iSOTP (Middleton, MandevilleNorden \& Hayes, 2009), and Inform Plus psychoeducation (Dervley et al., 2017). Social Science Protocols, September 2018, 1-12.

http://dx.doi.org/10.7565/ssp.2018.2648 
Nevertheless, it has been consistently noted that IIOC offenders represent a heterogeneous group, who display wide variation in their sexual preoccupation with IIOC and their motivation to offend. Finkelhor (2009) has also pointed out that a fundamental problem with prevention policy in the criminal justice system is that it is largely based on stereotypical (and often mistaken) characterisation of sex offender populations. The evidence of diversity in the population of men in possession of IIOC indicates that a single strategy for deterrence based on individual characteristics is likely to be inadequate.

The Situational Crime Prevention model (Cornish \& Clarke, 2003) offers a framework for considering environmental factors related to deterrence, whereby crime prevention requires increasing effort and risks associated with committing the crime, while reducing the rewards. This could be applied to viewing IIOC by implementing strategies that reduce the opportunities for accessing images of child abuse and exploitation, and making the activity less rewarding and riskier. For example, one relevant deterrence strategy is the removal of child abuse images from the Internet (e.g., Microsoft's Photo DNA), particularly as high levels of Internet use and easy access to material, are indicated as risk factors in Internet offending (Babchishin, Hanson \& VanZuylen, 2015). Similarly, blocking efforts by Google and Microsoft have resulted in a 67\% drop in the past year in web-based searches for IIOC compared with no blocking activities from Yandex, who demonstrated no commensurate drop in IIOC searches (Steel, 2015). This situational prevention approach considers the whole population rather than focusing solely on known offenders, while also moving away from assumptions about paedophilic tendencies and deviant subgroups. It also acknowledges the fact that interest in sexual media depicting children is probably much higher than is reflected in the offender population (see, for example, Dombert et al., 2016). The interaction between the person and environment may also be key to deterrence (Clarke, Ribisl, Runyan \& Runyan, 2013; Wortley, 2013), where the immediate environment plays a fundamental role in initiating an individual's behaviour and shaping its course. For some offenders, image-related offences are not solitary activity but occur within a social context both in relation to website searching (Westlake \& Bouchard, 2016) as well as P2P networks (Wolak, Liberatore \& Levine, 2014) which may provide a good indication of for whom (and how) deterrents may be targeted.

The target of deterrence efforts may not be the viewer of IIOC or the Internet as a platform for hosting images, but children and young people, and the wider general public, through education initiatives. Although there is limited evidence for such programmes (Jones, Mitchell \& Walsh, 2014), education is a recurrent theme in promoting Internet safety (Moreno, Egan, Bare, Young \& Cox, 2013; Wurtele \& Kenny, 2016). Education of children is unlikely to have a large, direct impact on the viewing of IIOC, though may help to decrease the availability of self-generated images. However, campaigns at the general public may increase the salience of legal sanctions to IIOC viewers, or may promote help seeking in existing or potential viewers, or others within both the offender's and the victim's immediate social environment to interrupt activity (for example, the Dunkelfeld Project in Germany; Beier et al., 2009).

What is clear from this brief consideration of deterrence to viewing online IIOC is that it can be conceptualised in a number of ways. Furthermore, the scope of these different facets of deterrence means that literature is likely to be dispersed across a range of disciplines. A lack of systematic review of this diverse literature creates difficulties in extrapolating future research and policy directions from the existing evidence. Furthermore, restricting a review to one discipline is likely to be of reduced value for policymakers, for whom a disciplinary lens may not be so important as identifying knowledge, evidence and implications. The authors therefore aim to review the available literature on deterrents to viewing IIOC, across a range of disciplines, and synthesise this diverse literature base, using a meta-narrative review 
methodology. This review is funded by the National Society for the Prevention of Cruelty to Children (NSPCC). Greenhalgh, Robert, Macfarlane et al. (2005) provide a comprehensive account of the process as the original developers of the review approach; however, there are currently very few examples of how the meta-narrative review has been applied and adapted for other research topics and questions. There are some published examples of reviews (e.g. Addis, Davies, Greene, MacBride-Stewart, Shepherd, 2009; Chinn \& Homeyard, 2016; Collins \& Hayes, 2010). MacDonald, O’Leary, Stockwell and Reist (2016) published a protocol, where they describe in detail their planned methods for a review of the harms and benefits associated with e-cigarettes and vapour devices, and adaptations to the original method. The aim of the present paper is to outline the protocol for the meta-narrative review of deterrents to viewing online IIOC, alongside a rationale for the authors' planned approach in conducting the review, and the anticipated challenges at each stage. It is hoped that the paper will provide information about the key decisions made by the authors and identify some of the challenges for future researchers.

\section{Methods/Design}

\subsection{Meta-narrative review approach}

The meta-narrative review approach was developed by Greenhalgh and colleagues to explore the spread of innovations within health services, having recognised that a traditional review was not well equipped to manage the emerging complex literature, where relevant research and commentary was dispersed across different areas. The full report is available as a book (Greenhalgh, Robert, Bate, Macfarlane \& Kyriakidou, 2005) and two other papers describe the main findings (Greenhalgh, Robert, Macfarlane, Bate \& Kyriakidou, 2004) and methodological challenges (Greenhalgh, Robert, Macfarlane et al., 2005). The diversity of the literature base, and inevitably the concepts and terminology used, leads to the potential for "silos" of knowledge where disciplinary boundaries remain. Greenhalgh, Robert, Macfarlane et al. (2005) describe this phenomenon with reference to Kuhn's (1962) theory of scientific revolutions, whereby research within paradigms (or disciplines, in this instance) follows particular conventions for investigating and understanding a subject. Thus, rather than ignore these boundaries, the meta-narrative review aims to examine the evolutions of research within traditions, how they have influenced the kinds of questions asked and the methodological approaches used to answer them (Greenhalgh, Wong, Westhorp \& Pawson, 2011). The reviewer also aims to examine the narratives within each discipline, then synthesise these narratives to finally create an overarching summary of findings. The approach was deemed appropriate for our review because, similarly, there has been an interest in exploring deterrence of online IIOC through the lenses of various science disciplines, including computing science and information technology, as well as within social sciences, criminology and law. There is also a substantial relevant "grey" literature. The meta-narrative review described in this paper was registered with PROSPERO [CRD42017067498], the International Prospective Register of Systematic Reviews. The systematic methodology of this meta-narrative review follows the six-phase protocol as outlined by Greenhalgh, Robert, Macfarlane et al. (2005). These are the planning phase, search phase, mapping phase, appraisal phase, synthesis phase, and recommendations phase.

\section{Planning phase}

The planning phase took place in December 2016 and has now been completed. At the beginning of this phase, a multidisciplinary advisory group of academics and practitioners was established, reflecting the anticipated relevant research traditions of this review, including criminology, psychology, medicine, offender intervention, management and 
industry. Subsequently, preliminary meetings were held with the advisory board and funder to agree the scope of the review and draw up an initial question and aims. This was a crucial step in the process, as it became clear that the review had the capacity to grow indefinitely if all tangentially relevant literature was incorporated. It was established between the research team and funder that the purpose of the review would be to identify and map out evidence, policies and theories of deterrents to viewing IIOC online, where deterrence would relate to IIOC viewers both known and unknown to law enforcement, and therefore include strategies for prevention, disruption, detection, desistance and treatment. It was decided not to include papers on other related behaviours (online grooming, for example), unless the authors specifically mentioned IIOC online and deterrence. As an initial scoping search identified literature across a wide range of disciplinary traditions and grey literature fields (e.g. social sciences, law, child protection, information technology), it was agreed that the inclusion criteria would be broad in terms of research tradition and source.

\section{Search phase}

The research team, advisory panel and funder also collaborated in the generation of key terms for the literature search. The initial list of search terms was necessarily broad to identify literature across different fields. A university librarian with expertise in literature searching was also consulted for advice in identifying appropriate terms and databases for the different academic disciplines. The search strategy was also devised to map onto the key criteria for inclusion on the review. These inclusion criteria were that: the paper focuses on viewing IIOC, in an online environment, and discusses deterrence in relation to this behaviour. The third of these criteria is a particular challenge to apply, because it is hard to determine how much discussion is sufficient and relies somewhat on subjective judgement. For this reason, all papers marked for inclusion will be considered by two members of the research team. The literature search will be completed in two phases, first within the academic literature, and second within the grey literature. Due to the resources of the research team, only items published in English will be included.

For the academic literature, a systematic literature search was designed to source scholarly articles, books and book chapters, conference papers, proceedings, dissertations, theses and reports. Although MacDonald et al. (2016) excluded theses and dissertations because they were not part of the public health dialogue, we decided to include these because they are often widely available in the public domain so had the potential to influence or contribute to a narrative, and may provide recent evidence for deterrence. However, editorials summarising other papers in a journal that do not contain new information will be excluded. Several academic databases were identified to best represent the diverse fields of study relevant to this review: PsycINFO, MEDLINE, EMBASE and CINAHL for medicine and psychology; ASSIA, Social Services Abstract, ERIC and Social Sciences Premium Collection for the social sciences, IBSS and Scopus for global and public health services and social work/care; Westlaw UK, Criminal Justice Database, PAIS and LexisNexis for law, policing, criminal justice, child protection and public policy; Inspec and Compendex for informatics/media studies, prevention science, computer science and information technology; and ETHOS, ProQuest Dissertations and Theses Global for dissertations and theses specifically. All database searches will be limited to articles written in the English language, and published between January 2000 and $16^{\text {th }}$ April 2017. The 17-year period allowed for the inclusion of early work conducted into offender deterrence and the viewing of child images, which would facilitate understanding of how each research tradition had evolved. Search terms will focus on online offending, relating to IIOC, with relevance to deterrence and will be combined using Boolean connectors.

The grey literature will also be searched systematically, but here we have had to be 
creative with identifying an appropriate strategy for identifying relevant material. A list of keywords will be entered into Google search engine, which accepts a maximum of 30 terms. These identified keywords overlap with those used in the academic search (e.g. deter*, child*, adolescen*, indecent image* pornograph* Internet). The first 100 pages of returns will be examined for inclusion. The main inclusion criteria are the same as for the academic literature, but will also exclude civil society advocacy pages, offender registers, vigilante pages or offender lists, sex trafficking (not Internet), conference announcements, and freedom of information requests. Secondly, a list of sixteen key organisations and stakeholders has been created, and their organisation webpages will be hand searched for relevant literature, as well as the NSPCC resource library.

In both searches, references lists will also be scrutinised for relevant papers, and the advisory panel consulted to highlight any key papers not previously identified. Due to the wide range of search terms across both academic and non-academic sources, it is anticipated that the sifting of articles will be a very time consuming process; nevertheless this will be performed by sequentially looking at the title, abstract and full text, with reasons for exclusion recorded at full text review.

\section{Mapping phase}

Following the literature searches, the literature will be mapped onto the various research traditions. The endpoint of this process is to identify the key narratives within the different research traditions, consider how deterrence is conceptualised and measured within each, and how each narrative has developed over time. To reach this point, an extraction sheet will be used to record the discipline of the authors, year of publication, article type, the way deterrence is defined, the methods of inquiry (if applicable), the main argument of the paper and whether the paper contains primary or secondary evidence of deterrence, number of citations. Within the non-academic literature, the labelling of documents by research discipline is less intuitive and the number of citations harder to track, and therefore the papers will be recorded according to source, such as a particular stakeholder group or type of organisation, and citations not recorded; other data extracted will be the same as for the academic literature. This will aid the authors in grouping together the literature according to discipline or stakeholder group, then clustering these into underlying research traditions (of which there may be more than one in the various disciplines). Those key references that were of seminal importance to the understanding of deterring IIOC within each tradition will also be highlighted, based on number of citations. As suggested in Macdonald et al. (2016), the findings at this point will be shared with the funding body and advisory panel, to show progress and emerging themes to the results.

\section{Appraisal phase}

For this fourth phase, the identified academic and grey literature will be critically appraised using appropriate techniques to evaluate each item for its validity and relevance to the review questions and the influence to the overall evidence-base; in each instance, this requires some flexibility in the review process and the need to move between the appraisal and mapping phases, as the contribution to each research tradition is also considered within the mapping phase. During the search and mapping phases, the relevance will be considered and papers will not be excluded at this stage on the basis of overall quality, as this could mean that useful "nuggets" of information can be lost (Pawson, 2006); in other words, the value of a paper may be broader than its (reasonably) objective methodological quality, if it stimulates further discussion and research. The appraisal phase also aims to assess how much the paper has influenced the overall conclusions of the review. It is in relation to this second aim that the appraisal of literature has caused the greatest level of discussion within the 
research team, prompting debate around the value of appraisal when it is already recognised that different research fields may have different understandings of a gold standard in research, and where in other ways the review methodology has attempted to be as inclusive as possible. The range of methods used and the fact that many papers will not be primary research also precludes the use of a detailed, universal appraisal tool. However, while it is tempting to avoid the issue of quality, the corollary of this argument is that there is anticipated high value to researchers, the funding body and other stakeholders of identifying the strength of evidence and its limitations. The research team made the following decisions about how to manage the issue of quality. As discussed in the mapping phase, it was agreed to record the number of citations, or reference in later non-academic works, as an indicator of the paper's influence, however, it is acknowledged that this may undervalue more recent work that is driving a body of research. The data extraction sheet developed in the mapping phase and the overarching narratives from the synthesis phase will be used to assess the strength of evidence within each identified narrative, by using appropriate rating tools, e.g. Scottish Intercollegiate Guidelines Network checklists (SIGN, 2015) to appraise studies reporting primary evidence of deterrents to viewing IIOC, by study design. The use of appraisal checklists for studies of particular design is consistent with the method outlined by Greenhalgh, Robert, Bate et al. (2005). From this exercise, the authors aim to evaluate the strength of evidence for each key deterrence narrative.

\section{Synthesis phase}

This phase aims to synthesise the diversity of academic and grey literature in form of a conceptual framework to represent the findings within and across the research traditions. Such a framework should capture the overarching narrative to highlight where disciplinary boundaries diverge, merge and create broader understandings of the literature (Jamal, Bertotti, Lorenc \& Harden, 2015). Therefore, researchers will attempt to explain or reconcile any contestations in the narratives, though contestations can also serve to drive further research questions (Greenhalgh, Robert, Macfarlane et al., 2005). The introduction to this paper briefly touched on models of deterrence, and these offer potential frameworks for understanding the narratives; nevertheless, the reviewers aim to be guided as far as possible by the emerging review results, rather than forcing them into a specific structure.

\section{Recommendations phase}

The sixth phase and final phase aims to summarise the overall message from the academic and grey literature through a process of reflection and multidisciplinary dialogue with the funder. A final draft with key recommendations for practice, policy and further research will be produced based on feedback and discussion with the advisory group and funder of this study (c.f. Greenhalgh et al., 2004), and peer review. This will draw on the publication standards for meta-narrative reviews developed by the RAMESES group (Wong, Greenhalgh, Westhorp, Buckingham, \& Pawson, 2013). The review will also be disseminated through various academic channels, such as conference presentations and publication in academic journals, as well as professional channels, including talks at policy-maker meetings and publication of the meta-narrative review on various professional online platforms. Based on the large number of stakeholders in this area, it is important that the reach of the final review is extensive, and the funder will be instrumental in this dissemination process.

\section{Discussion}

There is currently little evidence about what works to deter people from viewing IIOC, and there has been no previous comprehensive review to address this question. The purpose 
of the review outlined here is to collate and synthesise literature across a wide range of academic and non-academic disciplines and sources, using a meta-narrative review methodology. The purpose of this protocol paper was to describe our planned approach to the review and the decisions made so far, in the hope that this will be of benefit to other researchers considering conducting this type of review. Within this discussion section, we consider further some of the challenges both anticipated and already encountered during the review process.

The first challenge is the resource implication of conducting a meta-narrative review. This relates directly to two of the underlying principles of the review, pragmatism and pluralism, outlined in Greenhalgh, Robert, Macfarlane et al. (2005). The principle of pragmatism reflects that it is not obvious what literature should or should not be included where literature is not neatly contained and draws upon an increasing number of "threads". Consequently, it is not reasonable to assume that study selection is entirely objective. After an initial agreement between the funder, research team and advisory panel of the review's intended scope, it was nevertheless clear that a consistent application of the inclusion criteria was not easy, where there are degrees of consideration to deterrence in a paper, or IIOC could be linked with other topics, such as sex trafficking or grooming, but equally may not be. We have chosen to manage this by developing a search strategy that is sufficiently broad to allow us to look for relevant content in papers not solely focused on online IIOC through a full text review. In relation to deterrence, it is also likely to be necessary to review the full text of the paper, though we will apply the criterion that the authors need to explicitly link the narrative to deterrence. The screening of papers for inclusion will also involve several members of the research team, so that any papers identified for inclusion by a team member will be reviewed again by another team member. As the number of returned papers is anticipated to be well over a thousand and the review of full text necessary for many of these items, other authors should not underestimate the amount of time and required for this phase, and may need to factor in a level of ambiguity and time for discussion in the application of inclusion criteria. Interestingly, Greenhalgh, Potts, Wong, Bark and Swinglehurst (2009) relied less on database searches because they had previously found it be somewhat inefficient and Collins and Hayes (2010) only reviewed abstracts; therefore other reviewers may wish to find ways to reduce the lengthy search and screening process.

The principle of pluralism draws attention to the phenomenon that there is unlikely to single theory in the identified literature, and that researchers need to try not to view familiar literature as more important than literature from other disciplines. We have responded to this note of caution by compiling a research team and advisory panel from different disciplines. In this case, there is an implication for costs of people and time, and monetary costs associated with holding meetings between individuals involved. In most instances then, researchers would be advised to seek appropriate and sufficient funding for the project. The involvement of the funding body can extend far beyond this however, and it has been our experience that engagement and interest from the funder is particularly valuable in managing the scope of the review and publicising it; we also anticipate the funder to play a significant role in dissemination of the project, particularly beyond academic networks to policymakers. The principle of peer review outlined by Greenhalgh, Robert, Bate et al. (2005) is a reminder that feedback from peers should be sought along the way, particularly where the literature is complex, and a group of researchers may start to develop similar thinking through working closely together. We will adhere to this principle through our advisory panel and identifying experts from different fields for a final peer review, but this places an additional demand on time to researchers and peer reviewers, that we would highlight to future researchers.

For all that the principle of pragmatism has been emphasised, it should be noted that the authors, who have prior experience in conducting the more traditional systematic review, 
have aimed to apply a systematic approach, transparency and rigour to the review process. Transparency is particularly important due to constructivist stance of the review, and its inherent subjectivity (Otte-Trojel \& Wong, 2016). As with a traditional systematic review, there is a detailed strategy for identifying relevant academic and non-academic literature through the use of databases and keywords, and drawing up a list of organisations to search for relevant documents, as well as relying on an Internet search engine. The methods of searching forwards and backwords will also be utilised, by looking at reference lists of included papers, and following citations. It is already clear that the management of literature will need to be systematic, and for this reference management software will be employed. Additionally, we will be rigorous in recording whether articles are excluded based on title, abstract or full text, with a reason recorded for any exclusions at full text review. In terms of transparency, we have published the review protocol with PROSPERO, will keep the advisory panel and funding body informed of developments and key decisions, and have presented here a more detailed account of the review protocol.

To conclude, the review described in this paper is seen as a timely venture, and a significant undertaking for establishing what is currently known or understood about the topic of deterrence to viewing IIOC across a vast realm of academic and non-academic sources. The intention is also to identify the strength of evidence for deterrence measures to help shape future policy and research.

\section{Declarations}

Availability of data and material: The datasets used and/or analysed during the current study are available from the corresponding author on reasonable request.

Funding: Funding was received from the National Society for the Prevention of Cruelty to Children (NSPCC).

Competing interests: The authors declare that they have no competing interests.

\section{References}

Addis, S., Davies, M., Greene, G., MacBride-Stewart, S., \& Shepherd, M. (2009). The health, social care and housing needs of lesbian, gay, bisexual and transgender older people: a review of the literature. Health and Social Care in the Community, 17, 647-65.

Babchishin, K.M., Hanson, R.K., \& Vanzuylen, H. (2015). Online child pornography offenders are different: a meta-analysis of the characteristics of online and offline sex offenders against children. Archives of Sexual Behavior, 44, 45-66.

BBC News (2016). Online child sex abuse investigation identifies 523 potential victims. Retrieved from http://www.bbc.co.uk/news/uk-scotland-36922820

Beier, K.M., Grundmann, D., Kuhle, L.F., Scherner, G., Konrad, A, \& Amelung, T. (2015). The German Dunkelfeld Project: a pilot study to prevent child sexual abuse and the use of child abusive images. The Journal of Sexual Medicine, 12, 529-542.

Beier, K.M., Neutze, J., Mundt, I.A., Ahlers, C.J., Goecker, D., \& Konrad, A., \& Schaefer, G.A. (2009). Encouraging self-identified pedophiles and hebephiles to seek professional help: first results of the Prevention Project Dunkelfeld. Child Abuse \& Neglect, 33, 545549. 
CEOP. (2012). A picture of abuse: a thematic assessment of the risk of contact child sexual abuse posed by those who possess indecent images of children. Retrieved from $\mathrm{http}: / /$ ceop.police.uk/publications/

Chinn, D., \& Homeyard, C. (2016). Easy read and accessible information for people with intellectual disabilities: is it worth it? A meta-narrative literature review. Health Expectations, 20, 1189-1200.

Clarke, M., Ribisl, K.M., Runyan, D., \& Runyan, C. (2013). A public health approach to addressing internet child sexual exploitation. In E. Quayle \& K.K.Ribisl (Eds.), Understanding and preventing online sexual exploitation of children (pp. 244-265). London: Routledge.

Collins, P.A., \& Hayes, M.V. (2010). The role of urban municipal governments in reducing health inequalities: a meta-narrative mapping analysis. International Journal for Equity in Health, 9, 13.

Cornish, D.B., \& Clarke, R.V. (2003). Opportunities, precipitators and criminal decisions: a reply to Wortley's critique of situational crime prevention. In M. J. Smith \& D.B. Cornish (Eds.), Theory for practice in situational crime prevention, crime prevention studies, 16 (pp. 111-124). Monsey, NY: Criminal Justice Press.

Dervley, R., Perkins, D., Whitehead, H., Bailey, A., Gillespie, S., \& Squire, T. (2017). Themes in participant feedback on a risk reduction programme for child sexual exploitation material offenders. Journal of Sexual Aggression, 23, 46-61.

Dombert, B., Schmidt, A.F., Banse, R., Briken, P., Hoyer, J., Neutze, J., \& Osterheider, M. (2016). How common is men's self reported sexual interest in prepubescent children? The Journal of Sex Research, 53, 214-223.

Elliot, I.A., \& Beech, A.R. (2009). Understanding online child pornography use: applying sexual offense theory to internet offenders. Aggression \& Violent Behavior, 14, 180-193.

Elliott, I. A., Beech, A.R., \& Mandeville-Norden, R. (2013). The psychological profiles of internet, contact, and mixed internet/contact sex offenders. Sexual Abuse: Journal of Research and Treatment, 25, 3-20.

Finkelhor, D. (2009). The prevention of childhood sexual abuse. Future Child, 19, 169-194.

Greenhalgh, T., Potts, H.W.W., Wong, G., Bark. P., \& Swinglehurst, D. (2009). Tensions and paradoxes in electronic patient record research: a systematic literature review using the meta-narrative method. The Milbank Quarterly, 87, 729-788.

Greenhalgh, T., Robert, G., Bate, P., Macfarlane, F., \& Kyriakidou, O. (2005). Diffusion of innovations in health service organisations: a systematic literature review. Oxford: Blackwell.

Greenhalgh, T., Robert, G., Macfarlane, F., Bate, P., \& Kyriakidou, O. (2004). Diffusion of innovations in service organizations: systematic review and recommendations. The Milbank Quarterly, 82, 581-629.

Greenhalgh, T., Robert, G., Macfarlane, F., Bate, P., Kyriakidou, O., \& Peacock, R. (2005). Storylines of research in diffusion of innovation: a meta-narrative approach to systematic review. Social Science and Medicine, 61, 417-430.

Greenhalgh, T., Wong, G., Westhorp, J., \& Pawson, R. (2011). Protocol-realist and metanarrative evidence synthesis: evolving standards. BMC Medical Research Methodology, $11,115$.

Henzey, M.J. (2011). Going on the offensive: a comprehensive overview of internet child pornography distribution and aggressive legal action. Appalachian Journal of Law, 11, 1123.

IWF (2016). Annual Report. Retrieved from https://www.iwf.org.uk/report/2016-annualreport 
Jamal, F., Bertotti, M., Lorenc, T., \& Harden, A. (2015). Reviewing conceptualisations of community: reflections on a meta-narrative approach. Qualitative Research, 15, 314-333.

Jones, L. M., Mitchell, K.J., \& Walsh, W.A. (2014). A content analysis of youth internet safety programs: are effective strategies being used? Durham, NH: Crimes Against Children Research Center (CCRC), University of New Hampshire.

Kuhn, T. (1962). The structure of scientific revolutions. Chicago: University of Chicago Press.

Leclerc, B., Feakes, J., Cale, J. (2015). Child sexual abuse in youth-oriented organisations: tapping into situational crime prevention from the offender's perspective. Crime Science, 4, 28.

MacDonald, M., O’Leary, R., Stockwell, T., \& Reist, D. (2016). Clearing the air: protocol for a systematic meta-narrative review on the harms and benefits of e-cigarettes and vapour devices. BioMed Central, 5, 85.

Middleton, D., Mandeville-Norton, R., \& Hayes, E. (2009). Does treatment work with internet sex offenders? Emerging findings from the Internet Sex Offender Treatment Programme (i-SOTP). Journal of Sexual Aggression, 15, 5-19.

Moreno, M.A., Egan, K.G., Bare, K., Young, H.N. and Cox, E.D. (2013). Internet safety education for youth: stakeholder perspectives. BMC public health, 13, 543.

Otte-Trojel, T. \& Wong, G. (2016). Going beyond systematic reviews: realist and metanarrative reviews. Studies in Health Technology \& Informatics, 222, 275-287.

Pawson, R. (2006). Digging for nuggets: how 'bad' research can yield 'good' evidence. International Journal of Social Research Methodology, 9, 127-142.

Quayle, E., \& Newman, E. (2015). The role of sexual images in online and offline sexual behaviour with minors. Current Psychiatry Reports, 17, 43.

Schuijer, J., \& Rossen, B. (1992). The trade in child pornography. IPT Forensics, 4. Retrieved from http://www.ipt-forensics.com/journal/volume4/j4_2_1.htm

Scottish Intercollegiate Guidelines Network (2015). SIGN 50: a guideline developer's handbook. Edinburgh: SIGN.

Seto, M.C., \& Eke, A.W. (2015). Predicting recidivism among adult male child pornography offenders: development of the Child Pornography Offender Risk Tool (CPORT). Law and Human Behavior, 39, 416-429.

Seto, M.C., Hanson, R.K., \& Babchishin, K.M. (2011). Contact sexual offending by men with online sexual offenses. Sexual Abuse: A Journal of Research and Treatment, 23, 124145.

Steel, C.M.S. (2015). Web-based child pornography: The global impact of deterrence efforts and its consumption on mobile platforms. Child Abuse \& Neglect, 44, 150-158.

Westlake, B. G., \& Bouchard, M. (2016). Liking and hyperlinking: Community detection in online child sexual exploitation networks. Social Science Research 59, 23-36.

Wolak, J., Finkelhor, D., \& Mitchell, K. (2012). Trends in arrests for child pornography production: the third national juvenile online victimisation study (NJOV-3). Durham, NH: Crimes against Children Research Center.

Wolak, J., Liberatore, M., \& Levine, B.N. (2014). Measuring a year of child pornography trafficking by U.S. computers on a peer to peer network. Child Abuse \& Neglect, 38, 342356.

Wong, G., Greenhalgh, T., Westhorp, G., Buckingham, J., \& Pawson, R. (2013). RAMESES publication standards: meta-narrative reviews. Journal of Advanced Nursing, 69, 9871004.

Wortley, R (2013). Situational prevention of child abuse in the new technologies. In E. Quayle \& K.K. Ribisl (Eds.), Understanding and preventing online sexual exploitation of children (pp. 188-203). London: Routledge. 
Wurtele, S.K., \& Kenny, M.C. (2016). Technology-related sexual solicitation of adolescents: a review of prevention efforts. Child Abuse Review, 25, 332-344. 